\title{
Clinical features of anterior uveitis caused by three different herpes viruses
}

\author{
Jun-ichi Sakai · Yoshihiko Usui • Jun Suzuki • Takeshi Kezuka • \\ Hiroshi Goto
}

Received: 10 October 2018/Accepted: 22 May 2019/Published online: 27 May 2019

(C) The Author(s) 2019

\begin{abstract}
Purpose To compare the clinical findings in patients with anterior uveitis (AU) caused by herpes simplex virus (HSV), varicella zoster virus (VZV), and cytomegalovirus (CMV).

Methods We retrospectively analyzed the clinical profiles of HSV-AU (14 patients), VZV sine herpete (ZSH-AU: 21 patients), and CMV-AU (17 patients) diagnosed by the detection of corresponding viral DNA in aqueous humor samples by polymerase chain reaction. Further, five patients with Posner-Schlossman (P-S) syndrome were selected as controls for CMV-AU.

Results Patients with CMV-AU were predominately male or older in age, and all cases were unilateral

The abstract of this paper was presented at the ARVO Annual Meeting Abstract June 2013, as a conference talk with interim findings. The poster's abstract was published in "Poster Abstracts" in Investigative Ophthalmology \& Visual Science June 2013, Vol. 54, 2909. http://iovs.arvojournals.org/article. aspx ?articleid $=2147647$. The accepted abstract is submitted as Supplementary Information.
\end{abstract}

Electronic supplementary material The online version of this article (https://doi.org/10.1007/s10792-019-01125-5) contains supplementary material, which is available to authorized users.

J. Sakai · Y. Usui $(\bowtie) \cdot$ J. Suzuki · T. Kezuka · H. Goto Department of Ophthalmology, Tokyo Medical University Hospital, 6-7-1 Nishishinjuku, Shinjuku-ku,

Tokyo 160-0023, Japan

e-mail: usuyoshi@gmail.com except for three patients with CMV-AU. Mutton-fat keratic precipitates (KPs) were found mostly in patients with HSV-AU and ZSH-AU. Severities of $\mathrm{AU}$ and viral load were the highest in $\mathrm{ZSH}-\mathrm{AU}$, followed by HSV-AU and CMV-AU. Iris atrophy was observed in HSV-AU (50\%) and ZSH-AU (76\%), with typical morphology of round type and sector type, respectively. In patients with $\mathrm{CMV}-\mathrm{AU}$, a ring-shaped $\mathrm{KP}$ was found in $53 \%$ patients, $76 \%$ of whom showed a decreased number of corneal endothelial cells. CMV was not detected in the aqueous humor of patients with typical P-S syndrome.

Conclusion Clinical findings of HSV-AU and VZVAU were similar; however, more inflammatory findings were observed in VZV-AU. Iris atrophy morphologically differed in HSV-AU and VZV-AU. Inflammatory findings in CMV-AU were mild, and clinical features of iritis differed from those of the two former groups. A difference in the etiology between CMV-AU and P-S syndrome was observed.

Keywords Anterior uveitis - Corneal endotheliitis . Cytomegalovirus · Herpes simplex virus · Varicella zoster virus

\section{Introduction}

Herpetic anterior uveitis (AU) is a well-recognized intraocular inflammatory disease that accounts for 
3-7\% of all uveitis cases in Japan [1-3]. It is the major cause of infectious AU, with herpes simplex virus (HSV) [4] and varicella zoster virus (VZV) [5, 6] identified as the most common causative agents by molecular techniques. Common clinical manifestations of AU include acute unilateral iridocyclitis, iris atrophy, elevation of intraocular pressure (IOP), and keratic precipitates (KPs), usually the mutton-fat type [7, 8]. HSV-AU and VZV-AU cases reportedly have good visual prognosis unless complicated by secondary glaucoma and corneal involvement [9].

Cytomegalovirus (CMV) has been recently identified as a causative agent of AU and corneal endotheliitis $[10,11]$. The virus has been detected in patients with AU not caused by HSV or VZV and in some patients with presumed Posner-Schlossman (P-S) syndrome or Fuchs heterochromic iridocyclitis $[12,13]$. A distinct feature of CMV-AU is coinshaped KPs and corneal endothelial cell (CEC) dysfunction [14, 15]. CMV infection in AU patients has been reported to reduce the number of CECs leading to bullous keratopathy [16]. Although the pathogenetic mechanism of CMV-AU remains unknown, it is believed to involve direct CMV infection of the CEC, because owl's eye-like morphology was observed by confocal microscopy $[17,18]$.

In patients with HSV iritis without keratitis and VZV iritis without skin eruption (zoster sine herpete) $[19,20]$, the diagnosis of herpetic uveitis can be difficult. Furthermore, CMV-AU is often misdiagnosed as $\mathrm{P}-\mathrm{S}$ syndrome. For the management of infectious uveitis, identification of the characteristic findings for early diagnosis and treatment is crucial to avoid irreversible ocular tissue damage. In this study, we investigated the clinical manifestations of uveitis caused by three different herpes viruses and attempted to clarify the distinctive features of each type of AU. We explored the pathogenesis of HSV-AU and VZVAU by investigating inflammation severity and prevalence of iris atrophy according to viral load and iris vasculature on indocyanine green angiography (ICGA). Moreover, we examined the difference between CMV-AU and P-S syndrome on the basis of clinical findings.

\section{Materials and methods}

Overall, 52 patients (55 eyes) with HVS-AU, VZV sine herpete (ZSH-AU), or CMV-AU diagnosed by the detection of corresponding viral DNA in aqueous humor samples by polymerase chain reaction (PCR), who attended the uveitis clinic of Tokyo Medical University Hospital, Japan, in 2001-2014, were included in this retrospective review. The study was approved by the Tokyo Medical University institutional review board, and informed consent was obtained from all participants included in the study.

Following suspected herpetic AU based on clinical findings, such as unilateral involvement, the presence of KP, and elevated IOP, aqueous humor samples were collected for viral DNA detection by PCR. Fourteen patients with HSV-AU, 21 patients with VZV sine herpete (ZSH-AU), and 17 patients with CMV-AU were included in this study, all with a confirmed diagnosis based on the detection of respective viral DNA by PCR. We selected consecutive patients with AU caused by HSV, VZV, and CMV. Of the patients with P-S syndrome who visited our hospital, we selected five who consented to anterior chamber puncture during the study period. Patients with dendritic keratitis and herpes zoster were excluded because the diagnosis was usually made clinically and PCR was not always performed. Quantitative PCR was performed for ten patients with HSV-AU, 15 patients with ZSH-AU, and 15 patients with CMV-AU [21]. None of the patients received anti-viral therapy before sample collection for PCR examination. In patients with bilateral disease, the more severely affected eye was selected for examination. Aqueous humor samples of five male patients with $\mathrm{P}-\mathrm{S}$ syndrome who provided consent for the examination (anterior chamber puncture) were also tested for PCR examination as a control group. Diagnosis of $\mathrm{P}-\mathrm{S}$ syndrome was based on clinical manifestation, including unilateral open-angle glaucoma with recurrent mild anterior chamber (AC) inflammation, the presence of some fine non-pigmented KPs on the central or inferior cornea, depigmentation in the trabecular area, and no anterior or posterior synechiae [22-24].

An ophthalmologic examination was performed when required based on the patients symptoms and included measurement of the best-corrected visual acuity, slit-lamp biomicroscopy, tonometry, and indirect ophthalmoscopy in all patients. Uveitis activity 
was assessed using the SUN criteria, as described previously [25]. Recurrence was assessed by the findings of iritis or corneal endotheliitis. Specular microscopy was performed for seven patients with HSV-AU, 13 patients with ZSH-AU, and 17 patients with CMV-AU. A CEC number under $1500 / \mathrm{mm}$ or $40 \%$ lower than the fellow eye was defined as CEC loss. Between patients with HSV-AU and ZSH-AU, we compared the severity of iritis using the number of inflammatory findings, such as ciliary injection, Descemet's membrane folds, corneal endotheliitis, mutton-fat KPs, iris atrophy, pupil distortion, and grading of AC cells $(1+-3+)$. An inflammation score was thereby generated (each finding was assigned 1 point; range 0-9; Fig. 1).

In five patients with ZSH-AU, ICGA was performed using a confocal scanning laser ophthalmoscope (F-10, Nidek, Gamagori, Japan). Clinical manifestations during the course of disease were compared on the basis of the causative herpes virus.

\section{Statistical analysis}

Statistical analysis was performed using SPSS ver. 22, and the statistical significance was set at $p<0.05$. One-way analysis of variance (ANOVA) was used to compare the mean age of the three patient groups, and Scheffe's $F$ test was performed as a post hoc test when ANOVA yielded a significant difference. Chi-square test and Fisher's exact test were used where

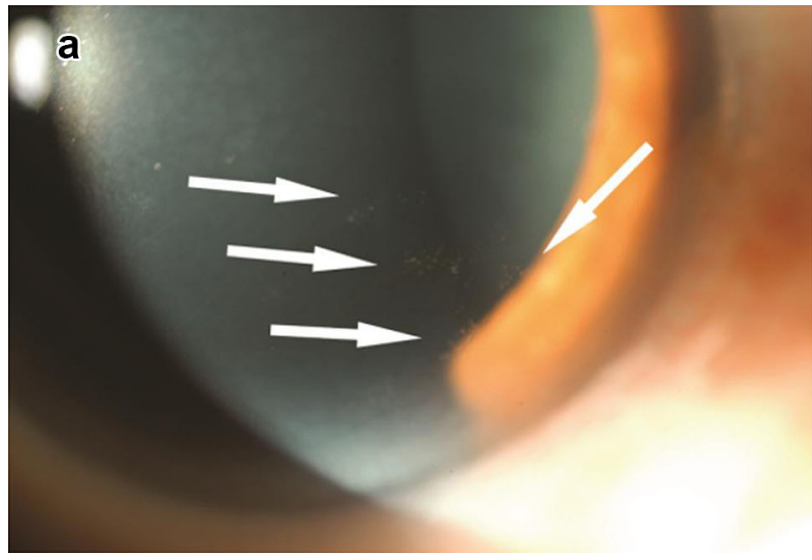

Fig. 1 Inflammation score in herpes simplex virus anterior uveitis (HSV-AU) and varicella zoster virus anterior uveitis (ZSH-AU) patients. The number of inflammatory findings, including ciliary injection, Descemet's membrane fold, corneal endotheliitis, mutton-fat keratic precipitates, iris atrophy, pupil appropriate to compare the demographic and clinical characteristics of the three groups. Residual analysis was performed to identify the specific difference when Chi-square test yielded a significant difference among the three groups. Mann-Whitney $U$ test was used to compare the severity between HSV-AU and ZSH-AU.

\section{Results}

Demographic characteristics of the three patient groups

The mean follow-up period for HSV-AU, ZSH-AU, and CMV-AU cases were 24.1, 18.3, and 30.2 months, respectively. Before diagnosis, one patient with $\mathrm{HSV}$ AU, one patient with ZSH-AU, and two patients with CMV-AU received cataract surgery. One patient with HSV-AU received cataract surgery and vitrectomy for retinal detachment, and one patient with CMV-AU received trabeculectomy and cataract surgery. The patients' demographic and clinical characteristics are summarized in Table 1 . The mean age at presentation was significantly different among the three groups (HSV-AU, ZSH-AU, and CMV-AU: 50.5, 51.1, and 70.0 years, $p=0.0003$, ANOVA), with a higher mean age in the CMV-AU group than that in the HSV-AU and ZSH-AU groups ( $p<0.01$ in both, Scheffe's $F$ test). The gender ratio was significantly different among the three groups (HSV-AU, ZSH-AU, and

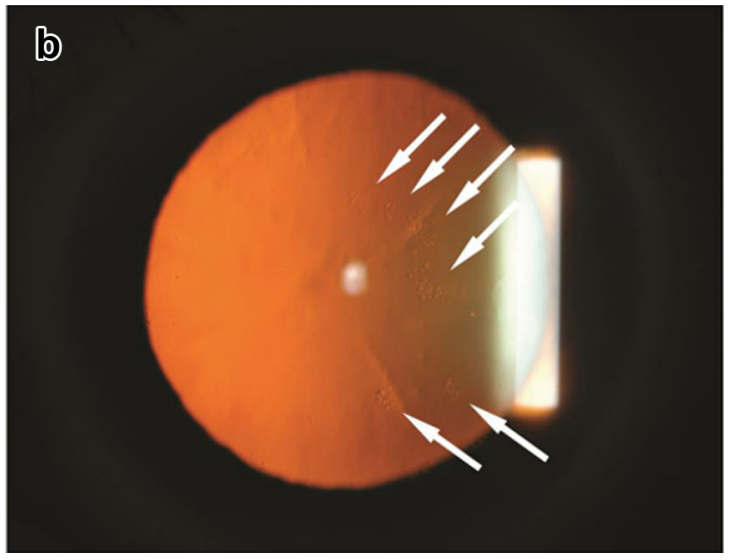

distortion, and cells in the anterior chamber (grading 1+ to 3+), between HSV-AU and ZSH-AU patients are compared. The average number is indicated by a horizontal black line. ${ }^{*} p=0.022$, Mann-Whitney $U$ test 
Table 1 Demographic and clinical background of the 52 patients studied

\begin{tabular}{|c|c|c|c|c|}
\hline & HSV-AU $(n=14)$ & ZSH-AU $(n=21)$ & CMV-AU $(n=17)$ & $p$ value \\
\hline Age; mean \pm SD (years) & $50.5 \pm 16.0$ & $51.1 \pm 16.8$ & $70.0 \pm 10.8^{\mathrm{a}}$ & $<0.01^{\mathrm{b}}$ \\
\hline Sex, $n(\%)$ & & & & $<0.01^{\mathrm{c}}$ \\
\hline Male & $5(36)$ & $6(29)$ & $13(76) * *$ & \\
\hline Female & $9(64)$ & $15(71)^{*}$ & $4(24)$ & \\
\hline Eye involvement & & & & $<0.05^{\mathrm{c}}$ \\
\hline Unilateral & $14(100)$ & $21(100)$ & $14(82)$ & \\
\hline Bilateral & $0(0)$ & $0(0)$ & $3(18)^{* *}$ & \\
\hline Visual acuity & & & & $0.25^{\mathrm{c}}$ \\
\hline$\geq 20 / 20$ & $7(50)$ & $5(25)$ & $4(24)$ & \\
\hline $20 / 40-20 / 25$ & $4(29)$ & $6(27)$ & $3(18)$ & \\
\hline$\leq 20 / 50$ & $3(21)$ & $10(48)$ & $10(58)$ & \\
\hline IOP elevation & $12(86)$ & $21(100)$ & 17 (100) & $0.06^{\mathrm{c}}$ \\
\hline Recurrence & $8(57)$ & $10(48)$ & $17(100)^{* *}$ & $<0.01^{\mathrm{c}}$ \\
\hline
\end{tabular}

Data are expressed as number of patients with percent in parenthesis, unless indicated otherwise

$H S V$ - $A U$ herpes simplex virus anterior uveitis, $Z S H-A U$ varicella zoster virus sine herpete, $C M V$ - $A U$ cytomegalovirus anterior uveitis, $I O P$ intraocular pressure

${ }^{*} p<0.05$, residual analysis; $* * p<0.01$, residual analysis

${ }^{\mathrm{a}} p<0.01$ versus HSV-AU and ZSH-AU, Scheffe's $F$ test

${ }^{\mathrm{b}} \mathrm{ANOVA}$

${ }^{\mathrm{c}} \chi^{2}$ test

CMV-AU: M/F 5/9, 6/15, and 13/4, $p=0.009$, Chisquare test); patients with CMV-AU were predominately male ( $p<0.01$, residual analysis). The prevalence of unilateral or bilateral disease was significantly different among the three groups $(p=0.038$, Chisquare test). All patients with HSV-AU and ZSH-AU had unilateral involvement, while three patients with CMV-AU had bilateral disease $(p<0.01$, residual analysis). Visual acuity at initial visit was diverse. Almost all patients in all three groups had elevated IOP (HSV-AU, ZSH-AU, and CMV-AU: $86 \%, 100 \%$, and $100 \%$, respectively). The recurrence rate was significantly different among the three groups ( $p=0.0018$, Chi-square test); it was the highest in the CMV-AU group $(100 \% ; p<0.01$, residual analysis) and approximately 50\% in the HSV-AU and $\mathrm{ZSH}-\mathrm{AU}$ groups. In patients with CMV-AU, seven $(41 \%)$ were followed with a presumptive diagnosis of P-S syndrome and eight (47\%) were followed with recurrent iritis and IOP elevation before being referred to our hospital.
Clinical features of corneal involvement

Either mutton-fat or fine non-granulomatous KPs were observed in all patients, but the predominant morphology differed among the patients in the three groups ( $p=0.00007$, Chi-square test; Table 2). Mutton-fat KPs were observed in many patients with HSVAU (93\%; $p<0.05$, residual analysis) and ZSH-AU (86\%; $p<0.05$, residual analysis), while fine nongranulomatous KPs were found in $71 \%$ of the patients with CMV-AU $(p<0.01$, residual analysis). Pigmented KPs developed during the course of disease in $57 \%$ of the patients with HSV-AU, $81 \%$ of the patients with ZSH-AU, and $82 \%$ of the patients with CMVAU, with no significant difference. Ring-shaped KPs (Fig. 2) were observed in patients with CMV-AU ( $p=0.00001$, Chi-square test) and were detected in $53 \%$ of the patients with CMV-AU $(p<0.01$, residual analysis).

There was a significant difference in the distribution of KPs (focal or diffuse) among the three groups ( $p=0.006$, Chi-square test). Diffuse large KPs were 
Table 2 Clinical features of corneal involvement

\begin{tabular}{|c|c|c|c|c|}
\hline & $\operatorname{HSV}-\mathrm{AU}(n=14)$ & ZSH-AU $(n=21)$ & CMV-AU $(n=17)$ & $p$ value $^{\mathrm{a}}$ \\
\hline Keratic precipitates (KPs) & $14(100)$ & $21(100)$ & $17(100)$ & \\
\hline Morphology of KPs & & & & $<0.01$ \\
\hline Mutton-fat & $13(93)^{* *}$ & $18(86)^{* *}$ & $5(29)$ & \\
\hline Fine non-granulomatous & $1(7)$ & $3(14)$ & $12(71)^{* *}$ & \\
\hline Pigmented KPs & $8(57)$ & $17(81)$ & $14(82)$ & 0.20 \\
\hline Ring-shaped KPs & $0(0)$ & $0(0)$ & $9(53)^{* *}$ & $<0.01$ \\
\hline Distribution of KPs & & & & $<0.01$ \\
\hline Focal & $2(14)$ & $8(38)$ & $12(71)^{* *}$ & \\
\hline Diffuse & $12(86)^{* *}$ & $13(62)$ & $5(29)$ & \\
\hline Corneal edema & $7(50)$ & $7(21)$ & $8(47)$ & 0.55 \\
\hline Descemet's membrane folds & $2(14)$ & $8(38)$ & $3(18)$ & 0.20 \\
\hline Corneal endotheliitis & $3(21)$ & $0(0)$ & $6(35)^{*}$ & $<0.05$ \\
\hline CECs loss ${ }^{\mathrm{b}}$ & $0(0)$ & $2(15)$ & $13(76)^{* *}$ & $<0.01$ \\
\hline
\end{tabular}

Data are expressed as number of patients with percent in parenthesis

$H S V$ - $A U$ herpes simplex virus anterior uveitis, $Z S H$ - $A U$ varicella zoster virus sine herpete, $C M V$ - $A U$ cytomegalovirus anterior uveitis, $C E C$ corneal endothelial cells

$* p<0.05$, residual analysis; $* * p<0.01$, residual analysis

${ }^{\mathrm{a}} \chi^{2}$ test

${ }^{\mathrm{b}}$ Specular microscopy was conducted in 7 patients with HSV-AU, 13 with ZSH-AU, and 17 with CMV-AU

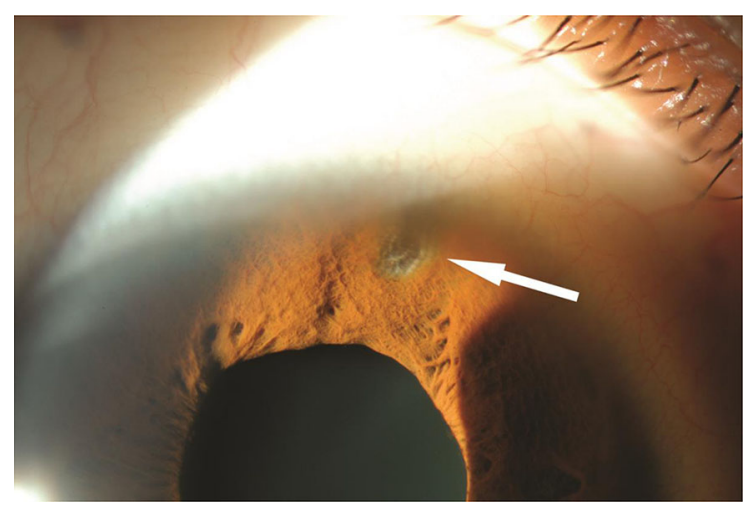

Fig. 2 Keratic precipitates in patients with cytomegalovirus anterior uveitis. a Representative slit-lamp photograph showing ring-shaped keratic precipitates on the endothelial surface (arrows). b Representative retro-illumination corneal photograph clearly demonstrating the ring-shaped keratic precipitates (arrows)

found in $86 \%$ of the patients with HSV-AU $(p<0.01$, residual analysis) and in $62 \%$ of the patients with ZSH-AU $(p>0.05)$, whereas small focal KPs were found in $71 \%$ of the patients with CMV-AU $(p<0.01$, residual analysis). The prevalence of clinically defined corneal endotheliitis was significantly different among the three groups ( $p=0.015$, Chi-square test) and was observed in six patients with CMV-AU (35\%; $p<0.05$, residual analysis). CEC loss was most frequently observed in the CMV-AU group (76\%; $p<0.01$, residual analysis), with a significant difference among the three groups (0/7, HSV-AU; $2 / 13$, ZSH-AU; and 13/17, CMV-AU cases tested; $p=0.0002$, Chi-square test).

Clinical features of conjunctiva, AC, and iris

The prevalence of ciliary injection and the cell number in the AC were significantly different among the three groups ( $p=0.00002$ and $p=0.041$, respectively, Chisquare test; Table 3). Ciliary injection was observed in almost all patients with HSV-AU (93\%) and ZSH-AU (95\%) but only a few patients with CMV-AU (35\%) ( $p<0.01$, residual analysis). All patients with HSVAU and ZSH-AU had grade $1+$ or higher cell numbers in the $\mathrm{AC}$, while most patients with $\mathrm{CMV}$ $\mathrm{AU}$ had $1+$ or lower cell numbers in the $\mathrm{AC}$ $(p<0.05$ and $p<0.01$, respectively, residual 
Table 3 Clinical features of conjunctiva, anterior chamber, and iris

\begin{tabular}{|c|c|c|c|c|}
\hline & HSV-AU $(n=14)$ & ZSH-AU $(n=21)$ & CMV-AU $(n=17)$ & $p$ value $^{\mathrm{a}}$ \\
\hline Ciliary injection & $13(93)$ & $20(95)^{* *}$ & $6(35)^{* *}$ & $<0.01$ \\
\hline Cells in AC & & & & $<0.05$ \\
\hline None & $0(0)$ & $0(0)$ & $2(12)^{* *}$ & \\
\hline Grade $1+$ & $8(57)$ & $10(48)$ & $14(82)^{*}$ & \\
\hline Grade $2+$ & $6(43)$ & $10(48)$ & $1(6)$ & \\
\hline Grade $3+$ & $0(0)$ & $1(4)$ & $0(0)$ & \\
\hline Iris atrophy & $7(50)$ & $16(76)^{* *}$ & $3(18)^{* *}$ & $<0.01$ \\
\hline Round & $7 * *$ & 3 & 0 & \\
\hline Sectoral & 0 & $13^{* *}$ & 0 & \\
\hline Diffuse & 0 & 2 & $3 * *$ & \\
\hline Pupil distortion & $6(43)$ & $17(81)^{* *}$ & $0(0)^{* *}$ & $<0.01$ \\
\hline
\end{tabular}

Data are expressed as number of patients with percent in parenthesis, or number of patients only

$H S V$ - $A U$ herpes simplex virus anterior uveitis, $Z S H-A U$ varicella zoster virus sine herpete, $C M V$ - $A U$ cytomegalovirus anterior uveitis, $A C$ anterior chamber

$* p<0.05$, residual analysis; $* * p<0.01$, residual analysis

a $\chi^{2}$ test

analysis). No cells were observed in the AC of two patients with CMV-AU, although these patients had a history of AC inflammation $(1+)$ before referral to our hospital.

Viral copy number in the $\mathrm{AC}$ was the highest in patients with $\mathrm{ZSH}-\mathrm{AU}\left(1.2 \pm 1.3 \times 10^{7}\right)$, followed by HSV-AU $\left(2.8 \pm 4.4 \times 10^{5}\right)$ and CMV-AU $\left(3.1 \pm 6.8 \times 10^{4}\right)$. The ratio of viral copy number in the three groups (HSV-AU:ZSH-AU:CMV-AU) was approximately 400:10:1. As several reports suggested that CMV was a causative agent for P-S syndrome $(12,13)$, we investigated the viral DNA of aqueous humor. In five patients with $\mathrm{P}-\mathrm{S}$ syndrome $(56.8 \pm 15.9$ years old $)$, herpes virus was not detected in the aqueous humor samples.

The prevalence and morphology of iris atrophy were different among the three groups $(p=0.0016$ and $2.7 \times 10^{-6}$, respectively, Chi-square test). The prevalence of iris atrophy was higher in patients with ZSH-AU (76\%; $p<0.01$, residual analysis) and less frequent in those with CMV-AU (18\%; $p<0.01$, residual analysis). Round-shaped iris atrophy (Fig. 3) was observed in all patients with HSV-AU $(p<0.01$, residual analysis), while patchy or sectoral iris atrophy (Fig. 4) was a typical feature in patients with ZSH-AU (13/21 cases; $p<0.01$, residual analysis). Diffuse iris atrophy was found in three patients with CMV-AU

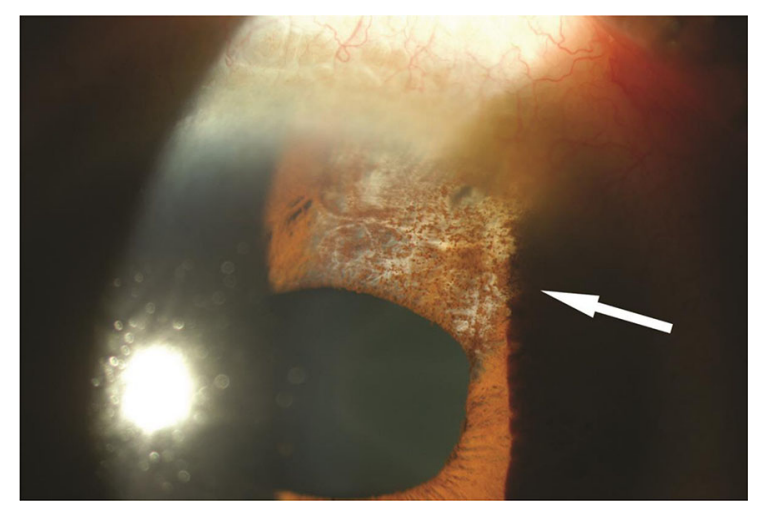

Fig. 3 Iris atrophy in patients with herpes simplex virus anterior uveitis. A representative slit-lamp photograph showing round-shaped iris atrophy (arrow)

( $p<0.01$, residual analysis). In these patients, iris transillumination and posterior synechiae were not observed. Similar to iris atrophy, the prevalence of pupil distortion was different among the three groups $\left(p=3.8 \times 10^{-6}\right.$, Chi-square test). Pupil distortion was seen in many patients with ZSH-AU (81\%; $p<0.01$, residual analysis), some patients with HSVAU (43\%), and no patient with CMV-AU $(p<0.01$, residual analysis). In the patients with iris atrophy or pupil distortion (HSV-AU, ZSH-AU, and CMV-AU: 9, 18, and 3 patients, respectively), only one patient 


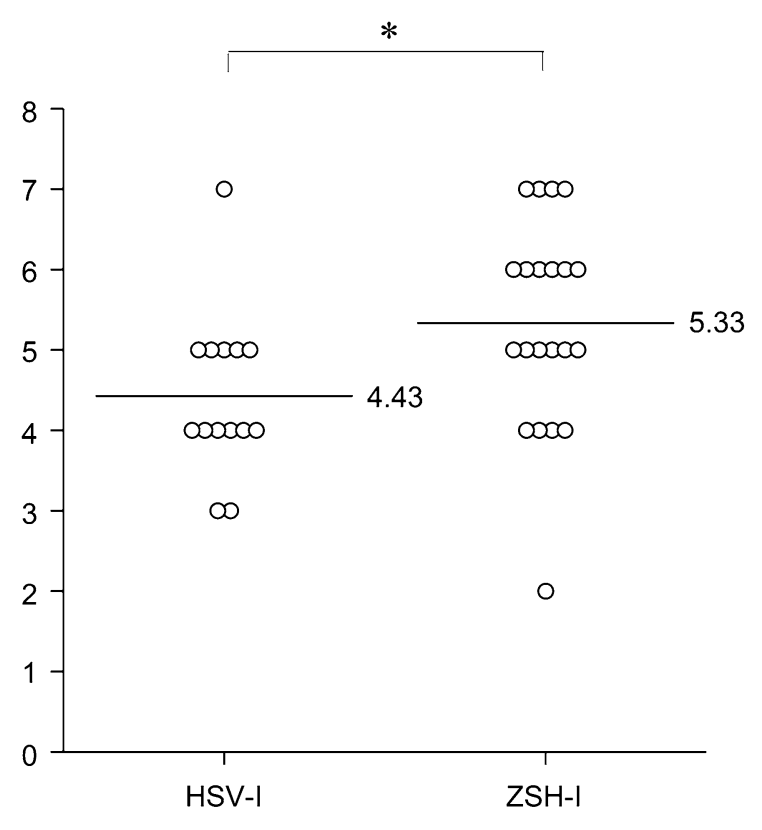

Fig. 4 Iris atrophy in patients with varicella zoster virus anterior uveitis. A representative slit-lamp photograph showing sectoral iris atrophy with distorted pupil (arrow)

with HSV-AU received intraocular surgery (cataract surgery).

Clinical findings and severity of HSV-AU and $\mathrm{ZSH}-\mathrm{AU}$

Herpetic iridocyclitis is classified into two groups. There were many common features with the clinical findings of HSV-AU and VZV-AU. Therefore, we compared these groups in order to better clarify their characteristics. Between HSV-AU and ZSH-AU, the viral copy number in the $\mathrm{AC}$ was significantly higher in the ZSH-AU group than in the HSV-AU group $\left(1.2 \pm 1.3 \times 10^{7}\right.$ vs. $2.8 \pm 4.4 \times 10^{5} ; p<0.01$, Mann-Whitney $U$ test). The severity of iritis between patients with HSV-AU and those with ZSH-AU was then compared. The number of inflammatory findings, such as ciliary injection, Descemet's membrane folds, corneal endotheliitis, mutton-fat KPs, iris atrophy, pupil distortion, and grading of AC cells $(1+$ to $3+)$, was calculated, and an inflammation score was evaluated (each finding was assigned 1 point; range 0-9; Fig. 1). As a result, the inflammation score was statistically higher in the ZSH-AU group $(5.33 \pm 1.28)$ than in the HSV-AU group $(4.33 \pm 1.02 ; p=0.022$, Mann-Whitney $U$ test $)$.
The prevalence of iris atrophy was higher in the ZSH-AU group (16/21 cases) than in the HSV-AU group (7/14 cases), with no statistical difference ( $p=0.110$, Fisher's exact test). However, the morphology was different between the two groups; roundshaped iris atrophy was higher in the HSV-AU group ( $p=0.029$, Fisher's exact test), and sectoral iris atrophy ( $p=0.0001$, Fisher's exact test) and pupil distortion were higher in the ZSH-AU group ( $p=0.025$, Fisher's exact test).

Iris ICGA was also performed to visualize the iris vasculature in five patients with $\mathrm{ZSH}-\mathrm{AU}$. While blood flow from the circulus arteriosus major to the circulus arteriosus minor of the iris via a radial vessel was observed, lack of vascular filling was detected corresponding with sectoral iris atrophy (Fig. 5).

\section{Discussion}

Herpetic uveitis is a major cause of AU, whereby HSV, VZV, and CMV are recognized as the causative agents. The present study focused on the clinical features of $\mathrm{AU}$ and clarified some similarities and differences among AU caused by HSV, VZV, and CMV. Greater awareness of the characteristic clinical features of each viral uveitis is crucial for early diagnosis and appropriate treatment, resulting in better prognosis.

In the present study, the frequent clinical findings of HSV-AU, ZSH-AU, and CMV-AU were subdivided into two groups on the basis of similar presentation. HSV-AU and ZSH-AU were shown to have similar clinical features, such as moderate-to-severe inflammation (ciliary injection and grade $2+$ or higher cell numbers in the AC), mutton-fat KP with diffuse distribution, iris atrophy, and pupil distortion. Conversely, CMV-AU tends to occur with recurrent mild iritis with focally distributed fine non-granulomatous KPs or ring-shaped KPs as the common clinical features. Moreover, patients with CMV-AU developed corneal endotheliitis accompanied with a reduced number of CECs.

Together with older age and patients being male, recurrent non-granulomatous mild iritis accompanied with a reduced number of CECs by corneal endotheliitis is considered typical clinical features of CMVAU. The above clinical findings are considered critical features of herpetic AU because they are similar to 

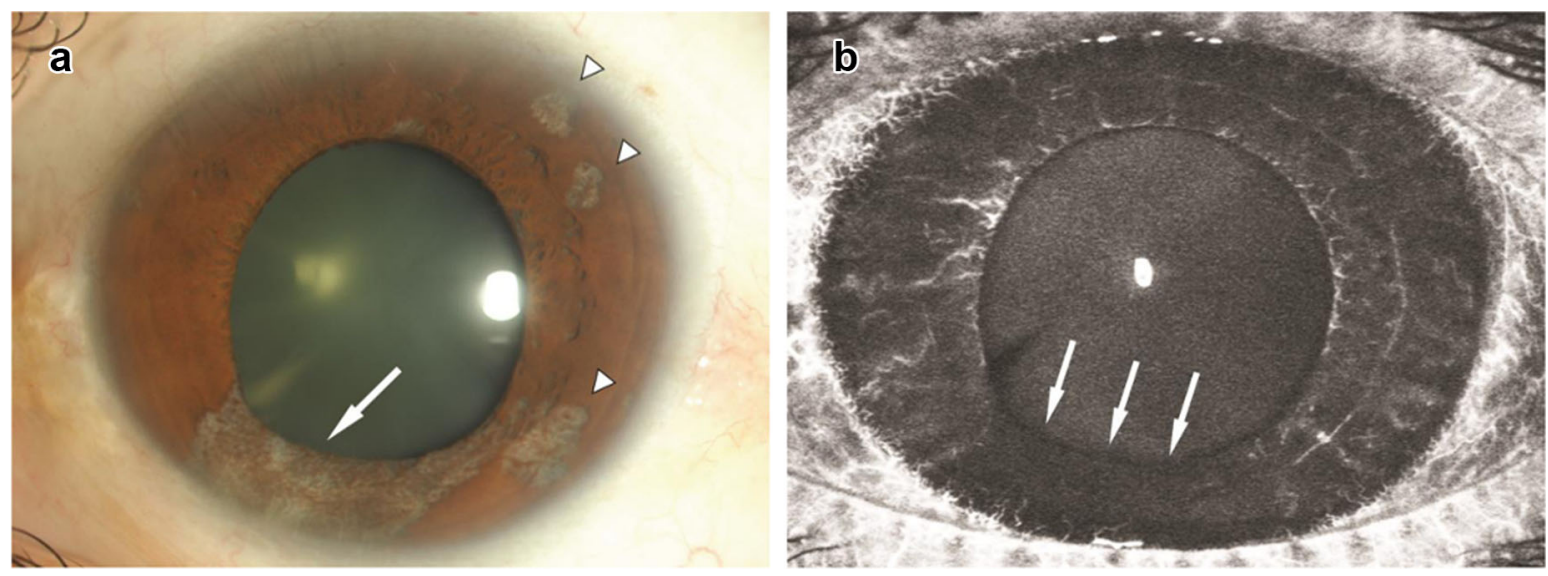

Fig. 5 Iris indocyanine green angiography in patients with ZSH-AU. a Sectoral (arrow) and round-shaped iris atrophy (arrowheads) were detected in patients with ZSH-AU. b Sectoral iris atrophy was clearly depicted as a loss of the vascular filling area (arrows)

those previously reported in a Japanese study conducted during the same period [26]. In this study, we examined the differences in the degree of ocular inflammation, the number of viral copies, the form of iris atrophy, and reductions in the number of CECs across three different types of herpes virus iritis through the latent and propagation forms of each virus.

In patients with $\mathrm{AU}, \mathrm{ZSH}$ had the highest viral copy number in the AC, followed by HSV and CMV; the same sequence order was also observed for the severity of AU. Intraocular inflammation was more severe in the ZSH-AU group than in the HSV-AU and CMV-AU groups, whereby patients with CMV-AU revealed quite mild inflammation compared to those with HSV-AU and ZSH-AU. In two patients with CMV-AU, no inflammatory cells were found in the AC during the observation periods, even though these patients had a history of iritis. After latent infection in sensory ganglia, HSV and VZV can reactivate and be transported to the axon terminal (anterograde transport), thereby enabling dissemination of the viruses into epithelial tissue, while latent infection of CMV is established in monocytes [27]. Along with chronic inflammation, monocytes migrate into the $\mathrm{AC}$ where dormant CMV in monocytes becomes activated and causes infection in CECs and the trabecular meshwork. Therefore, the pathogenesis of CMV-AU and that of HSV-AU and ZSH-AU are believed to be different. Regarding HSV and VZV, reactivated VZV reportedly migrates to not only sensory ganglion but also satellite cells around sensory ganglion neurons, such as Schwann cells, thereby resulting in the infection of neurons including a large portion of the dermatome, presenting as the herpes zoster rash [28]. In contrast, HSV reactivation is restricted to individual neurons within the ganglion. These differences in viral transmission of HSV, VZV, and CMV may affect the different viral copy numbers in the $\mathrm{AC}$ and the resulting severity of AU.

Regarding the pathology of iris atrophy, the prevalence and morphology was also related to the different routes of viral transmission and viral copy number. Patients with ZSH-AU frequently presented with iris atrophy, and the typical morphology was patchy or sectoral iris atrophy. Sectoral iris atrophy specific to ZSH-AU occurs due to ischemic change in the iris, and its pathogenesis is different from the pathogenesis which causes scalloped and well-defined iris atrophy observed in HSV-AU [29]. Types of iris atrophy are different in the infection caused by the three herpes viruses, and the differences are thought to be caused by the difference in the mode of transmission of these viruses. That is, in VZV, where a large amount of reactivated viruses go downward in axon as entangling Schwann cells around nerve fibers, viruses are transmitted to the long posterior ciliary artery which runs adjacent to the axon and occlusive vasculitis in the iris occurs along with sectoral iris atrophy. Different from scalloped and well-defined iris atrophy, it was confirmed by the iris IGGA that sectoral iris atrophy occurs due to occlusion of vascular in the iris; thus, sectoral iris atrophy becomes characteristic findings. Moreover, diffuse iris atrophy occasionally observed in $\mathrm{CMV}-\mathrm{AU}$ is not accompanied with dyscoria and is 
predicted to be caused by persistent ocular hypertension rather than being caused by tissue damage by viruses. In this study, nonperfusion area (NPA) corresponded with the sectoral iris atrophy area but not with the round area on ICGA. In contrast, a correlation between VZV viral copy number and damage to the iris (iris atrophy and pupil distortion) has been reported in patients with herpes zoster ophthalmicus and $\mathrm{ZSH}$ [30]. However, our study indicated that there was no correlation between morphology of iris atrophy and viral copy numbers despite the findings of Kido et al. [30]. While HSV and VZV are transmitted from the trigeminal ganglion to the eye via the trigeminal nerve, VZV virus invasion may also occur from the long ciliary nerve to the long posterior ciliary arteries for spreading to the circulus arteriosus major of the iris. Based on the finding of high VZV viral load that infected the eye, sectoral iris atrophy was deemed a characteristic finding in patients with ZSH-AU.

Even though most patients showed corneal endotheliitis and CEC loss, CMV-AU exhibited milder inflammation than HSV-AU and ZSH-AU. While diffuse iris atrophy was found in three patients with CMV-AU, iris transillumination and posterior synechiae were not observed. Therefore, iris atrophy could be associated with persistently high IOP rather than AC inflammation. These findings suggest that the main focus of inflammation induced by CMV involves CECs, and iritis may develop as a secondary reaction, possibly explaining why AC inflammation found in patients with CMV-AU was weak or sometimes absent.

In the present study, CMV was not detected in the aqueous humor of patients with typical P-S syndrome. $\mathrm{P}-\mathrm{S}$ syndrome is a disease with typically good prognosis and is characterized by mild inflammation in the AC: transient IOP elevation, small-to-medium, non-pigmented KP on the central and inferior cornea, and depigmentation of the trabecular area [22-24]. Although several reports have suggested that CMV is a causative agent of $\mathrm{P}-\mathrm{S}$ syndrome [12, 13], CMV-AU and typical P-S syndrome should be considered carefully. Although ciliary injection and pigmented KPs were found in 35\% (6/17 patients) and 82\% (14/ 17 patients), respectively, in CMV-AU, none of these findings were noted in patients with $\mathrm{P}-\mathrm{S}$ syndrome [22]. In addition to ring-shaped KPs and a decreased number of CEC, the presence of ciliary injection and pigmented KPs may be useful findings to differentiate between $\mathrm{CMV}-\mathrm{AU}$ and $\mathrm{P}-\mathrm{S}$ syndrome.
Depigmentation of the trabecular area observed in the affected eye of patients with $\mathrm{P}-\mathrm{S}$ syndrome is also an important finding to distinguish between the two. However, the pathogenesis of CMV-AU remains unclear. Additional investigations on CMV-AU are needed to clarify the pathogenesis of this disease, including the predominance of male patients.

\section{Conclusion}

In summary, while AU caused by various herpes viruses shares common clinical features, each type of AU exhibits distinct characteristic findings. Although a small number of patients were examined, these results may help with the diagnosis of herpetic AU and to clarify the pathomechanisms of each type of AU.

Acknowledgements This work was supported by a Grand-inAid for Scientific Research (C) 16K11330 from the Ministry of Education, Culture, Sports, Science, and Technology of Japan.

\section{Compliance with ethical standards}

Conflict of interest The author reports no conflicts of interest in this work.

Research involving human participants and/or animals For this type of study, formal consent was not required.

Informed consent Informed consent was obtained from all participants included in the study.

Open Access This article is distributed under the terms of the Creative Commons Attribution 4.0 International License (http:// creativecommons.org/licenses/by/4.0/), which permits unrestricted use, distribution, and reproduction in any medium, provided you give appropriate credit to the original author(s) and the source, provide a link to the Creative Commons license, and indicate if changes were made.

\section{References}

1. Sakai JI, Usui Y, Sakai M, Yokoi H, Goto H (2010) Clinical statistics of endogenous uveitis: comparison between general eye clinic and university hospital. Int Ophthalmol 30:297-301. https://doi.org/10.1007/s10792-009-9336-5

2. Goto H, Mochizuki M, Yamaki K, Kotake S, Usui M, Ohno S (2007) Epidemiological survey of intraocular inflammation in Japan. Jpn J Ophthalmol 51:41-44. https://doi.org/ 10.1007/s10384-006-0383-4

3. Ohguro N, Sonoda KH, Takeuchi M, Matsumura M, Mochizuki M (2012) The 2009 prospective multi-center 
epidemiologic survey of uveitis in Japan. Jpn J Ophthalmol 56:432-435. https://doi.org/10.1007/s10384-012-0158-Z

4. Ohashi Y, Yamamoto S, Nishida K, Okamoto S, Kinoshita S, Manabe R, Hayashi K (1991) Demonstration of herpes simplex virus DNA in idiopathic corneal endotheliopathy. Am J Ophthalmol 112:419-423

5. Yamamoto S, Tada R, Shimomura Y, Pavan-Langston D, Dunkel EC, Tano Y (1995) Detecting varicella-zoster virus DNA in iridocyclitis using polymerase chain reaction: a case of zoster sine herpete. Arch Ophthalmol 113:1358-1359. https://doi.org/10.1001/archopht.1995. 01100110018009

6. Yamamoto S, Pavan-Langston D, Kinoshita S, Nishida K, Shimomura Y, Tano Y (1996) Detecting herpesvirus DNA in uveitis using the polymerase chain reaction. Br J Ophthalmol 80:465-468

7. Tugal-Tutkun I, Otuk-Yasar B, Altinkurt E (2010) Clinical features and prognosis of herpetic anterior uveitis: a retrospective study of 111 cases. Int Ophthalmol 30:559-565. https://doi.org/10.4274/tjo.92053

8. Jap A, Chee SP (2011) Viral anterior uveitis. Curr Opin Ophthalmol 22:483-488. https://doi.org/10.1097/icu. 0b013e32834be021

9. Sungur GK, Hazirolan D, Yalvac IS, Ozer PA, Aslan BS, Duman S (2010) Incidence and prognosis of ocular hypertension secondary to viral uveitis. Int Ophthalmol 30:191-194. https://doi.org/10.1007/s10792-009-9305-z

10. van Boxtel LA, van der Lelij A, van der Meer J, Los LI (2007) Cytomegalovirus as a cause of anterior uveitis in immunocompetent patients. Ophthalmology 114:1358-1362. https:// doi.org/10.1016/j.ophtha.2006.09.035

11. Chee SP, Bacsal K, Jap A, Se-Thoe SY, Cheng CL, Tan BH (2007) Corneal endotheliitis associated with evidence of cytomegalovirus infection. Ophthalmology 114:798-803. https://doi.org/10.1016/j.ophtha.2006.07.057

12. Chee SP, Bacsal K, Jap A, Se-Thoe SY, Cheng CL, Tan BH (2008) Clinical features of cytomegalovirus anterior uveitis in immunocompetent patients. Am $\mathrm{J}$ Ophthalmol 145:834-840. https://doi.org/10.1016/j.ajo.2007.12.015

13. Chee SP, Jap A (2008) Presumed fuchs heterochromic iridocyclitis and Posner-Schlossman syndrome: comparison of cytomegalovirus-positive and negative eyes. Am J Ophthalmol 146:883-889. https://doi.org/10.1016/j.ajo. 2008.09.001

14. Koizumi N, Suzuki T, Uno T, Chihara H, Shiraishi A, Hara Y, Inatomi T, Sotozono C, Kawasaki S, Yamasaki K, Mochida C (2008) Cytomegalovirus as an etiologic factor in corneal endotheliitis. Ophthalmology 115:292-297. https:// doi.org/10.1016/j.ophtha.2007.04.053

15. Kandori M, Inoue T, Takamatsu F, Kojima Y, Hori Y, Maeda N, Tano Y (2010) Prevalence and features of keratitis with quantitative polymerase chain reaction positive for cytomegalovirus. Ophthalmology 117:216-222. https:// doi.org/10.1016/j.ophtha.2009.06.059

16. Yamauchi Y, Suzuki J, Sakai J, Sakamoto S, Iwasaki T, Usui M (2007) A case of hypertensive keratouveitis with endotheliitis associated with cytomegalovirus. Ocul Immunol Inflamm 15:399-401. https://doi.org/10.1080/ 09273940701486795

17. Shiraishi A, Hara Y, Takahashi M, Oka N, Yamaguchi M, Suzuki T, Uno T, Ohashi Y (2007) Demonstration of “owl's eye" morphology by confocal microscopy in a patient with presumed cytomegalovirus corneal endotheliitis. Am J Ophthalmol 143:715-717. https://doi.org/10.1016/j.ajo. 2006.11.026

18. Kobayashi A, Yokogawa H, Higashide T, Nitta K, Sugiyama K (2012) Clinical significance of owl eye morphologic features by in vivo laser confocal microscopy in patients with cytomegalovirus corneal endotheliitis. Am J Ophthalmol 153:445-453. https://doi.org/10.1016/j.ajo. 2011.07.026

19. Stavrou P, Mitchell SM, Fox JD, Hope-Ross MW, Murray PI (1994) Detection of varicella-zoster virus DNA in ocular samples from patients with uveitis but no cutaneous eruption. Eye (Lond) 8(Pt 6):684-687. https://doi.org/10.1038/ eye. 1994.169

20. Schwab IR (1997) Herpes zoster sine herpete. A potential cause of iridoplegic granulomatous iridocyclitis. Ophthalmology 104:1421-1425. https://doi.org/10.1016/S01616420(97)30121-3

21. Sugita S, Shimizu N, Watanabe K, Oka N, Yamaguchi M, Suzuki T, Uno T, Ohashi Y (2008) Use of multiplex PCR and real-time PCR to detect human herpes virus genome in ocular fluids of patients with uveitis. $\mathrm{Br} \mathrm{J}$ Ophthalmol 92:928-932. https://doi.org/10.1136/bjo.2007.133967

22. Shazly TA, Aljajeh M, Latina MA (2011) PosnerSchlossman glaucomatocyclitic crisis. Semin Ophthalmol 26:282-284. https://doi.org/10.3109/08820538.2011. 605821

23. Posner A, Schlossman A (1953) Further observations on the syndrome of glaucomatocyclitic crises. Trans Am Acad Ophthalmol Otolaryngol 57:531-536

24. Posner A, Schlossman A (1958) Syndrome of unilateral recurrent attacks of glaucoma with cyclitic symptoms. Arch Ophthal 39:517-535. https://doi.org/10.1001/archopht. 1948.00900020525007

25. Jabs DA, Nussenblatt RB, Rosenbaum JT (2005) Standardization of Uveitis Nomenclature (SUN) Working Group. Standardization of uveitis nomenclature for reporting clinical data. Results of the First International Workshop. Am J Ophthalmol 140:509-516

26. Takase H, Kubono R, Terada Y, Imai A, Fukuda S, Tomita M, Miyanaga M, Kamoi K, Sugita S, Miyata K, Mochizuki M (2014) Comparison of the ocular characteristics of anterior uveitis caused by herpes simplex virus, varicellazoster virus, and cytomegalovirus. Jpn J Ophthalmol 58:473-482. https://doi.org/10.1007/s10384-014-0340-6

27. Taylor-Wiedeman J, Sissons JG, Borysiewicz LK, Sinclair JH (1991) Monocytes are a major site of persistence of human cytomegalovirus in peripheral blood mononuclear cells. J Gen Virol 72:2059-2064. https://doi.org/10.1099/ 0022-1317-72-9-2059

28. Oxman MN (2010) Zoster vaccine: current status and future prospects. Clin Infect Dis 51:197-213. https://doi.org/10. $1086 / 653605$

29. Marsh RJ, Easty DL, Jones BR (1974) Iritis and iris atrophy in Herpes zoster ophthalmicus. Am J Ophthalmol 78:255-261. https://doi.org/10.1016/0002-9394(74)900865

30. Kido S, Sugita S, Horie S, Miyanaga M, Miyata K, Shimizu N, Morio T, Mochizuki M (2008) Association of varicella zoster virus load in the aqueous humor with clinical 
manifestations of anterior uveitis in herpes zoster ophthalmicus and zoster sine herpete. $\mathrm{Br} \mathrm{J}$ Ophthalmol 92:505-508. https://doi.org/10.1136/bjo.2007.125773
Publisher's Note Springer Nature remains neutral with regard to jurisdictional claims in published maps and institutional affiliations. 